ISSN 1991-8631

Original paper

http://indexmedicus.afro.who.int

\title{
Contamination spatio-temporelle d'origine hydrique de l'oued Boufekrane dans la région de Meknès-Tafilalt (Maroc)
}

\author{
Majdouline LARIF ${ }^{1 *}$, Abdelmajid SOULAYMANI ${ }^{2}$, Mohamed HNACH $^{3}$ et \\ Azzedine EL MIDAOUI ${ }^{1}$ \\ ${ }^{I}$ Laboratoire des Procédés de Séparation, Faculté des Sciences, Université Ibn Tofail, Kénitra, Maroc. \\ ${ }^{2}$ Laboratoire de Génétique et Biométrie, Faculté des Sciences, Université Ibn Tofail, Kénitra, Maroc. \\ ${ }^{3}$ Laboratoire de Spectrométrie des Matériaux et Archéologie, Faculté des Sciences, Université Moulay Ismail, \\ Meknès, Maroc. \\ *Auteur correspondant, E-mail : majdoulinelarif@yahoo.com; GSM : (212) 0665415516
}

\section{RESUME}

L'objectif de la présente étude est d'identifier l'origine et le degré de la contamination bactérienne instantanée des eaux courantes de l'oued Boufekrane de la région Meknès-Tafilalet (Maroc). Pour cela des analyses bactériologiques ont été réalisées sur sept sites de prélèvement qui couvrent l'oued Boufekrane. Le suivi spatio-temporel des coliformes totaux (CT), coliformes fécaux (CF), streptocoques fécaux (SP), anaérobie clostridium sulfito-réducteurs (ACSR) et flore mésophile totale (FMAT) montre que la charge bactérienne de ces germes dépasse largement les normes marocaines et les directives de l'organisation mondiale de la santé (OMS). Il a révélé au cours de la période 2009-2010, une variabilité spatio-temporelle des quatre saisons en temps sec et en temps de pluie par l'influence importante du type d'occupation des sols et des phénomènes de lessivage sur la composition bactériologique de l'oued. L'analyse en composantes principales (ACP) montre une forte corrélation spatio-temporelle entre les stations-mois et la contamination microbienne. Le test de Duncan montre un effet hautement significatif $(p<0,001)$ entre la localisation des stations et les saisons. Il nous permet de conclure que les sites sont caractérisés en amont par une forte pollution en nitrates et une faible contamination en matière fécale, alors qu'en aval, les eaux de l'oued sont caractérisées par une forte contamination fécale et par une faible pollution en nitrates.

(C) 2013 International Formulae Group. All rights reserved.

Mots clés : Oued Boufekrane, bactériologie, nitrates, contamination fécale, effet sanitaire, ACP.

\section{INTRODUCTION}

La vallée de l'oued Boufekrane, étalée sur près de 900 ha, est considérée comme le poumon vert de la ville de Meknès et l'épine dorsale faisant l'articulation entre deux espaces urbains particuliers : la médina de Meknès et la nouvelle ville. Au Maroc, les basses eaux, surtout en été, limitent la capacité d'autoépuration des cours d'eau, ce qui les rend vulnérables à la pollution (Fagrouch et al., 2011 ; Hadou-Sanoun et al., 2012).

Le milieu aquatique reçoit des rejets d'origine animale ou anthropique et industrielle soit par les déchets d'abattoirs en viande rouge (panse, cornes, viandes saisies) (Denis et al., 2011) ou bien par les pesticides d'origine agricole, qui proviennent surtout par ruissellement des zones de grandes cultures, de polycultures ou des zones d'élevage en

(C) 2013 International Formulae Group. All rights reserved.

DOI : http://dx.doi.org/10.4314/ijbcs.v7i1i.14 
mutation vers la culture intensive. Ceux-ci affectent les eaux souterraines mais aussi superficielles par une dégradation résultant des apports provenant des nappes, des ruissellements et des réseaux de drainage $(\mathrm{Xu}$ et al., 2011). Les modèles de contamination par les pesticides dans les rivières ont été décrits tout au long des années dans un certain nombre de flux à travers le monde (Konstantinou et al., 2006; Gilliom, 2007 ; Botta et al., 2012). Cependant, en milieu urbain l'utilisation des pesticides pourrait être d'une certaine importance en raison de plusieurs facteurs tels que l'imperméabilité des surfaces urbaines et l'absence de sensibilisation des utilisateurs urbains. (Blanchoud et al., 2004, 2007; Wittmer et al., 2010).

De ce fait, la transmission d'une maladie infectieuse fait intervenir un agent infectieux, un sujet réceptif et une voie d'introduction. Dans le cas des infections d'origine hydrique, les agents responsables de la contamination de l'eau sont les hommes et les animaux (Hartemann, 2004 ; Larif et al., 2013).

Le groupe des coliformes joue le rôle d'indicateur de pollution et de contamination fécale (Servais et al., 2009). En conséquence, il y a un risque de contamination des eaux de surface par des agents pathogènes d'origine hydrique responsables d'épidémies (Hebert et Legare, 2000 ; Toilabiya et al., 2012). Les salmonelles sont en général considérées comme les pathogènes les plus virulents en provoquant des maladies comme: fièvres typhoïdes, salmonelloses systémiques, gastroentérites, toxi-infections alimentaires. Humains et animaux peuvent éliminer dans les selles des salmonelles non seulement en cas de maladie mais aussi en tant que porteurs asymptomatiques. Les salmonelles peuvent donc être présentes dans l'eau des égouts agricoles et domestiques, les eaux douces, y compris les eaux potables et les nappes phréatiques.

Selon l'OMS, $80 \%$ des maladies qui affectent la population de la planète sont liées en partie à l'insuffisance de l'évacuation des matières fécales. Ces maladies sont donc, la cause d'un taux élevé de mortalité des populations des pays en voie de développement. La transmission des maladies d'origine hydrique dépend de trois facteurs : l'agent, l'environnement et l'individu (Rodier, 2009).

Dans cette étude, nous nous sommes proposés de suivre l'évolution saisonnière du degré de pollution de l'oued Boufekrane et d'identifier les stations qui sont à l'origine de la contamination. Ceci permettrait de contribuer à la mise en place du contrôle (à court terme) et à l'élaboration (à long terme) d'un système d'épuration adéquat en vue d'améliorer la qualité de ces eaux.

\section{MATERIEL ET METHODES}

\section{Echantillonnage}

Notre choix a été porté sur sept stations étalées sur le bassin versant de l'oued Boufekrane, en considérant le taux d'urbanisation élevé de ce bassin, de l'importance hydrologique des affluents et de l'accessibilité aux cours d'eaux. Ces eaux sont utilisées par pompage pour l'irrigation de surfaces importantes et pour le lavage des légumes au bord de l'oued. Les prélèvements des échantillons de l'amont (source témoin Ain Maarouf S1 vers l'aval S7 (Figure 1) ont été effectués d'une façon instantanée et mensuelle à 9 heures du matin au milieu de la rivière avec de la verrerie stérilisée, conservée à $4{ }^{\circ} \mathrm{C}$ et immédiatement transportée au laboratoire pour les analyses bactériologiques selon les normes d'AFNOR.

\section{Analyses bactériologiques}

L'analyse bactériologique a porté sur les germes indicateurs de pollution qui regroupent les bactéries hétérotrophes aérobies mésophiles tels que: Coliformes Totaux (CT) et Coliformes Fécaux (CF) (milieu de culture gélosé au Tergitol (TTC). Streptocoques Fécaux (SF) (milieu de culture Gélosé Slatetz et Bartley).

$$
\text { Pour les bactéries Anaérobies }
$$
clostridium sulfito-réducteurs (ACSR) le dénombrement est réalisé après incorporation 
d'un ml d'échantillon dilué dans un milieu sulfite polymixine sulfadiazine (SPS). Pour la flore mésophile aérobie totale FMAT, $1 \mathrm{~mL}$ de chaque dilution a été étalée en surface dans de la gélose nutritive acide comme milieu de culture PCA (Plate Count Agar) (Hemedia, M091).

La recherche éventuelle des salmonelles a été faite seulement au mois d'août pour les stations avals S3. S6 et S7 car ces dernières sont plus exposées à la pollution selon la méthode de Rodier (1996).

\section{Analyse chimique}

Le $\mathrm{pH}$ a été déterminé par le $\mathrm{pH}$-mètre (HANNA HI 120). La température a été mesurée sur place par un thermomètre graduée. Les concentrations des nitrates $\left(\mathrm{NO}_{3}{ }^{-}\right)$ont été mesurées au moyen d'un spectrophotomètre par la méthode de salysilate (Rodier, 2009). La Figure 4 indique les moyennes spatiales de la température de l'eau et du $\mathrm{pH}$.

\section{Analyse statistique}

Les données recueillies ont été saisies et soumises à des analyses statistiques à savoir: l'analyse de variance par le test de FISHER, la comparaison multiples des moyennes par le test de DUNCAN et l'Analyse en Composantes Principales (ACP) pour déceler la liaison entre les différentes variables, et variables-individus.

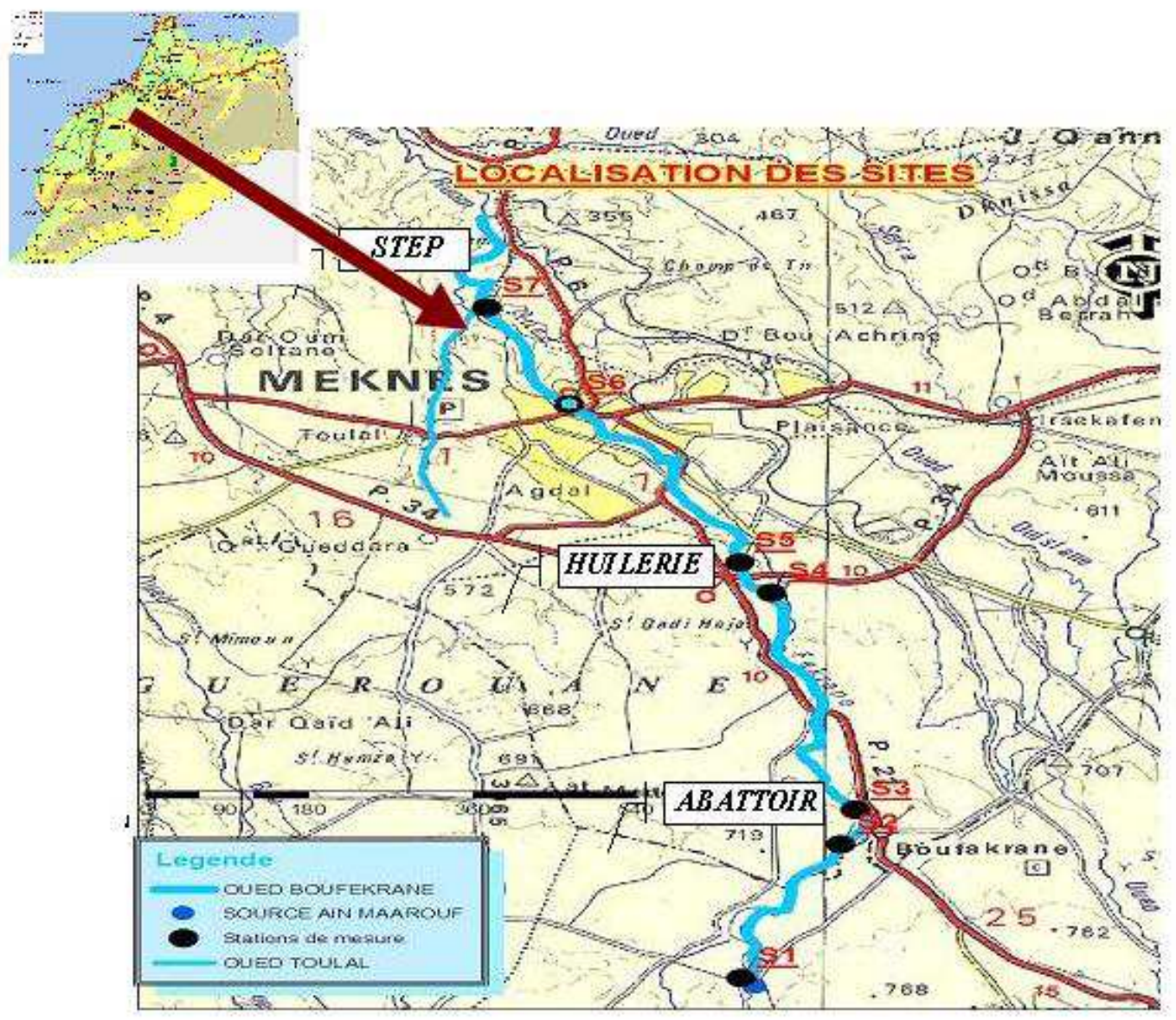

Figure 1 : Localisation graphique des sites d'études de l'oued Boufekrane. 


\section{RESULTATS}

Les résultats de l'ANOVA à un seul critère de classification effet station d'une part et effet saison d'autre part sur la répartition des coliformes totaux (CT), des coliformes fécaux (CF), les streptocoques fécaux (SF), l'anaérobies clostridium sulfito-réducteurs (ACSR), la flore mésophile aérobie totale à 37 ${ }^{\circ} \mathrm{C}$ (FMAT) et des nitrates de l'oued Boufekrane sont illustrés respectivement sur les Figures 2 et 3. Les lettres distinctes (a, b, c, d) désignent des différences au seuil de probabilité $1 \%$ (test de Duncan).

\section{Analyse temporelle}

Les résultats relatifs à la relation temporelle (en confondant les stations) en période (2009-2010) des indicateurs de la contamination fécale, des nitrates et de la flore mésophile aérobie totale sont indiqués suivant la Figure 2.

L'analyse de variance montre que la charge bactérienne en CT et en SF varie de manière très significative entre les quatre saisons ( $p<0,001$; pour les $\mathrm{CT}=6,91$ et pour les

$\mathrm{SF}=7,426)$ à $1 \%$ avec le test de Fisher $\left(\mathrm{F}_{\mathrm{CT}}=\right.$ $13,137, \mathrm{~F}_{\mathrm{SF}}=19,098, \mathrm{~F}_{\mathrm{CF}}=19,401, \mathrm{~F}_{\mathrm{FMAT}}=$ 23,706 et $\mathrm{F}_{\mathrm{ACSR}}=13,265$ )

La comparaison multiple des moyennes par le test de DUNCAN permet de classer les saisons en quatre groupes distincts pour les CT et les SF pendant uniquement l'été et l'hiver (Figure 2), tandis que pour les CF, FMAT et ACSR, l'analyse de variance indique des différences hautement significatives à $(1 \%)$ entre les saisons. La comparaison multiple des moyennes des CF, FMAT et ACSR dévoile respectivement des différences considérables sauf en automne et au printemps où elles sont similaires (Figure 2).

L'analyse descriptive, nous indique que les plus fortes concentrations sont obtenues respectivement plus en été, printemps, automne puis en hiver.

Pour les nitrates, la concentration la plus élevée est obtenue pendant la période hivernale qui est de $4,487 \mathrm{mg} / \mathrm{L}$ et en automne de $3,612 \mathrm{mg} / \mathrm{L}$. Ces concentrations restent faibles au printemps avec $2,162 \mathrm{mg} / \mathrm{L}$ puis en été de 3,087 mg/L (Figure 2(f)). L'analyse de variance montre une différence hautement significative (la valeur de $\mathrm{p}$ étant $\leq 0,0019$, avec $\left.\mathrm{F}_{\mathrm{NO} 3}=9,467\right)$. La comparaison multiple des moyennes indique des différences significatives entre les quatre saisons (Figure 2).

\section{Analyse spatiale}

Les résultats relatifs à la relation spatiale (en confondant les saisons) en période (2009-2010) des indicateurs de la contamination fécale, des nitrates et de la flore mésophile totale sont indiqués dans la Figure 3.

La variation spatiale des moyennes en amont de la station Ain Maarouf pour les CT, CF, SF, ACSR, FMAT dévoile respectivement de faibles concentrations à partir de 2,$139 ; 1,038 ; 0,985,0,742$ jusqu'à 1,048 unités $\log _{10}$ (UFC/100 mL). Ces concentrations augmentent de manière significative vers l'aval et les valeurs sont également respectivement de 4,$757 ; 4,203$; 4,$438 ; 1,935$ à 4,032 unités $\log _{10}$ (UFC/100 $\mathrm{mL})$. L'analyse de variance montre des différences hautement significative (à $1 \%$ ) entre les stations pour toutes les charges $\left(\mathrm{F}_{\mathbf{C T}}\right.$ $=13,137, \mathrm{~F}_{\mathrm{CF}}=19,401, \mathrm{~F}_{\mathrm{SF}}=19,098, \mathrm{~F}_{\mathrm{FMAT}}=$ 23,706 et $\left.F_{\mathrm{ACSR}}=13,265\right)$. Ces différences significatives sont confirmées par la comparaison multiple des moyennes par un gradient croissant de l'amont vers l'aval de l'oued Boufekrane, pour tous les groupes de bactéries étudiés (Figure 3).

Les teneurs en nitrates suivent un gradient décroissant de 4,74 à $1,59 \mathrm{mg} / \mathrm{L}$, Les valeurs obtenues sont conforment aux normes de l'OMS qui sont de $50 \mathrm{mg} / \mathrm{L}$. L'analyse de variance montre une différence hautement significative à $(1 \%$ o $)\left(\mathrm{F}_{\mathrm{NO} 3}=8,216\right)$ entre les stations (Figure 3 (f)).

\section{DISCUSSION}

La variation spatiale des moyennes de l'amont source Ain Maarouf pour les CT, CF, SF, ACSR, FMAT dévoilent respectivement de faibles concentrations qui augmentent de 
manière significative vers l'aval; L'analyse de variance a montré des différences hautement significatives (à 1\%o) entre les stations pour toutes les charges.Cette pollution pourrait s'expliquer par les rejets domestiques qui sont véhiculées à partir d'un réseau d'assainissement situé entre les stations S2 et S3 à l'aval de la commune Boufekrane (Genin et al., 2003 ; Kacar, 2011; Denis et al., 2011). Pour le tronçon S3 et S4, il y a une diminution des concentrations: ce qui explique le phénomène d'auto-épuration.

Tableau 1: Origine de la pollution selon le rapport coliformes fécaux/streptocoques fécaux $(\mathrm{R}=\mathrm{CF} / \mathrm{SF})$.

\begin{tabular}{llllllll}
\hline $\mathbf{R}=$ CF/SF & S1 & S2 & S3 & S4 & S5 & S6 & S7 \\
\hline Eté & 1,17 & 0,37 & 0,07 & 0,73 & 0,74 & 0,98 & 0,77 \\
Autumne & 2,17 & 0,77 & 1,05 & 1,08 & 0,34 & 1,08 & 1,16 \\
Hiver & 2,55 & 1,37 & 1 & 1 & 0,33 & 1 & 1 \\
Printemps & 0,92 & 1,71 & 0,8 & 0,92 & 0,92 & 0,76 & 0,24
\end{tabular}

$\mathrm{R}<0,7$ Principalement ou entièrement d'origine animale, $\mathrm{R}$ compris entre 0,7 et 1 mixte à prédominance animale, $\mathrm{R}$ compris entre 1 et 2 origine incertaine, $R$ compris entre 2 et 4 mixte à prédominance humaine, $R>4$ source exclusivement humaine.

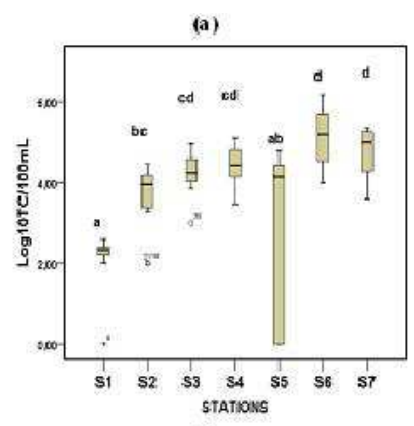

(d)

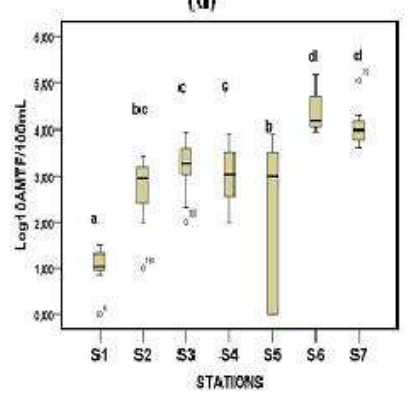

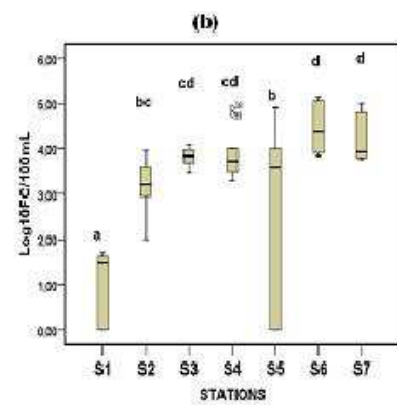

(e)

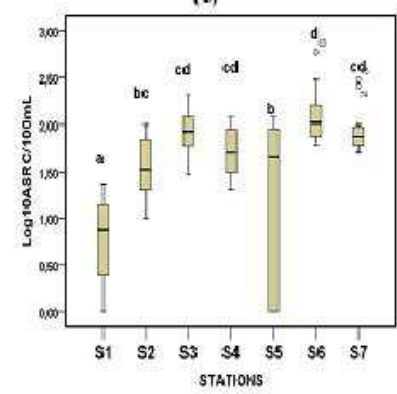

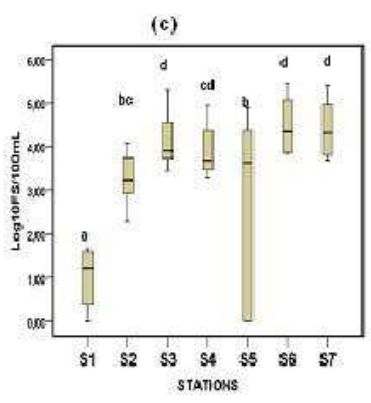

(i)

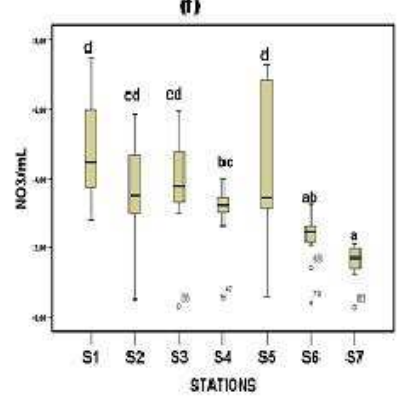

Figure 2 : Valeurs moyennes temporelles des abondances cellulaires exprimées en $\log _{10}$ (UFC/100 $\mathrm{mL}$ ) pour les coliformes totaux $(\mathrm{CT})$, coliformes fécaux $(\mathrm{CF})$, streptocoques fécaux (SF), anaérobies clostridium sulfito-réducteurs (ACSR), la flore mésophile aérobie totale à $37{ }^{\circ} \mathrm{C}$ (FMAT), et des nitrates $\left(\mathrm{NO}_{3}^{-}\right)$en $\mathrm{mg} / \mathrm{L}$. 

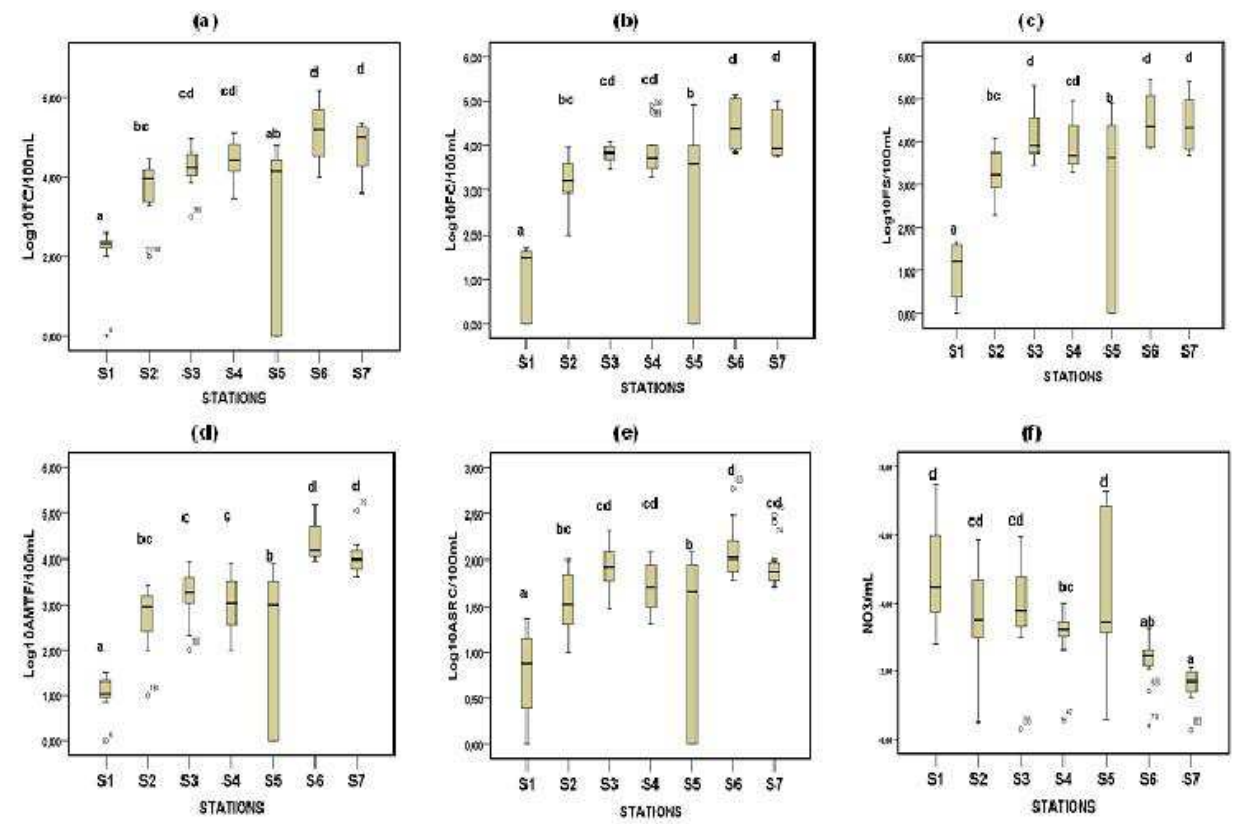

Figure 3 : Valeurs moyennes spatiales des abondances cellulaires exprimées en $\log _{10}$ (UFC/100 $\mathrm{mL}$ ) pour les coliformes totaux (CT), coliformes fécaux (CF), streptocoques fécaux (SF), anaérobies clostridium sulfito-réducteurs (ACSR), la flore mésophile aérobie totale à $37{ }^{\circ} \mathrm{C}$ (FMAT), et des nitrates $\left(\mathrm{NO}_{3}^{-}\right)$en $\mathrm{mg} / \mathrm{L}$.

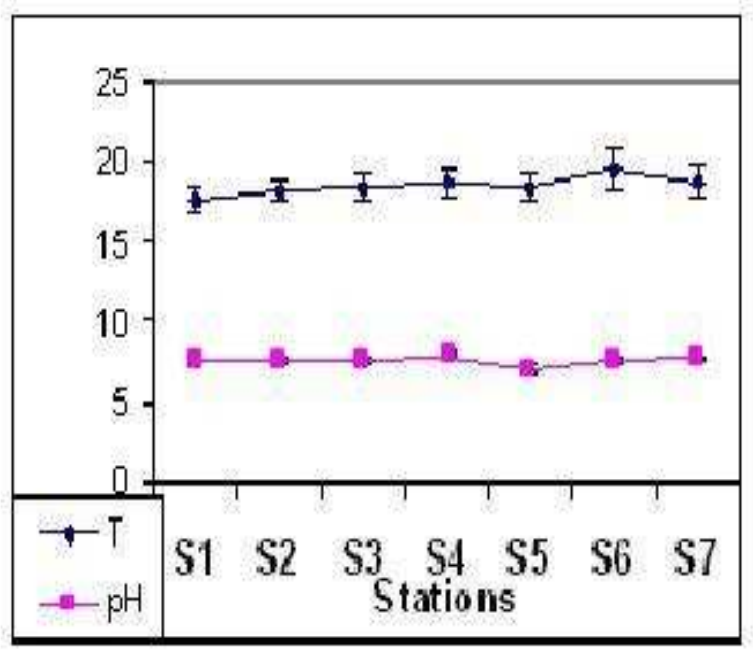

Figure 4 : Moyennes spatiales de la température de l'eau et du pH. 


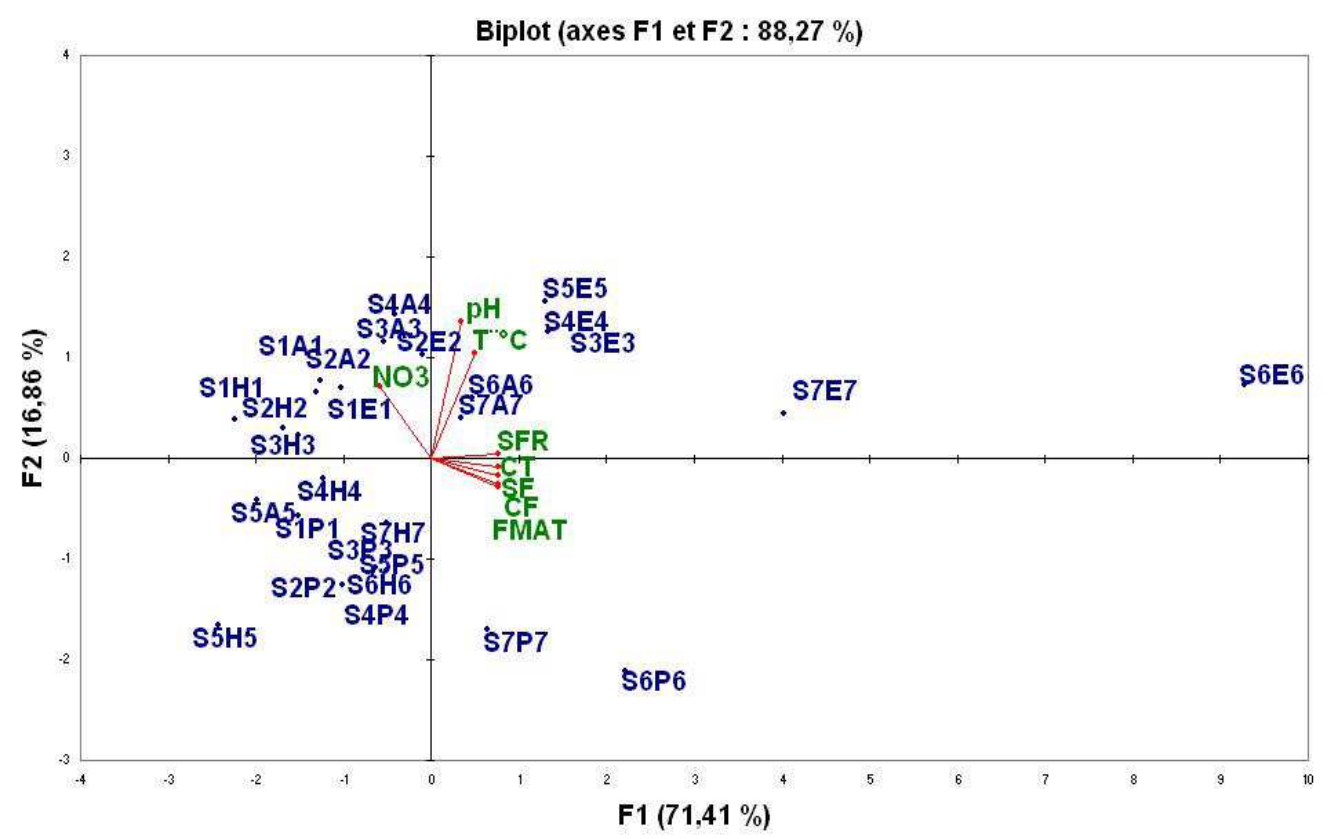

Figure 5 : Diagramme cartésien indiquant la corrélation entre les variables (paramètres bactériologiques et physiques) et les individus (stations- mois).

Pour la station S5, il y a absence de bactéries, car en période oléicole, il y a déversement des margines par les moulins à huile d'olive. Cependant les margines ont un caractère acide par la présence de polyphénols et d'acides gras qui sont défavorables au développement des microorganismes (Ranalli et al., 1991; Achak et al., 2008).

La variation temporelle des moyennes en été et en hiver sont uniquement pour les CT et SF et pour les moyennes en CF, FMAT et ACSR dévoile respectivement des différences considérables sauf en automne et au printemps où elles sont similaires aux plus fortes.

Les plus fortes concentrations sont obtenues en été. On pourrait l'expliquer par l'augmentation de la température et la durée d'ensoleillement pendant la période d'étiage au niveau des stations avale S5 et S6. Ces facteurs favorisent la multiplication des bactéries et leurs enrichissements par les phénomènes physiques adsorption, activation biologique, dilution, dispersion et sédimentation (Rodier 1996). Ils sont peu ou pas pathogènes, sont révélateurs de contamination fécale et entraînent par leur abondance la présomption de contamination plus dangereuse (Figarella et al., 2001 ; Kacar, 2011).

A l'observation, les CF sont supérieurs aux SF car les CF sont influencées par les facteurs abiotiques édaphiques et climatiques du milieu naturel (Aboulkacem et al., 2007 ; Cherbi et al., 2008).

Pour les bactéries ACSR, elles sont considérées comme des germes fécaux telluriques. Leur mise en évidence peut ne pas être attribuée car elle n'a aucune spécificité d'origine fécale. Dans une telle optique d'interprétation il y a intérêt à ne pas chercher que les espèces les plus susceptibles d'être d'origine fécale, c'est le cas en particulier de Clostridium perfringens (Rodier et al., 1996). Les Clostridium perfringens sont des bâtonnets anaérobies, gram (+), sporulant et qui réduisent les sulfites en sulfures en 24 à 48 heures (PNUE/OMS, 1977). Elles survivent dans les sédiments, ce qui permet de déceler 
une pollution ancienne ou intermittente (Rodier et al., 1996). La flore mésophile aérobie totale (FMAT) est utilisée comme un indicateur de pollution global. Elle englobe l'ensemble de microorganismes capables de se multiplier à l'air aux températures moyennes, surtout à une température optimale de croissance située entre 25 et $40{ }^{\circ} \mathrm{C}$. La FMAT nous a renseigné aussi bien sur la microflore autochtone que sur la microflore allochtone apportée par la pollution des eaux de l'oued Boufekrane (Rodier, 2009).

On remarque que d'après la Figure 5, les plus hautes valeurs des CT, CF, SF, ARSR et FMAT sont enregistrées en été et au printemps, ce qui nous confirme qu'il y a une forte corrélation entre la température, le $\mathrm{pH}$ de l'eau de rivière et le taux d'abattement des bactéries.

Pour les moyennes spatiales de la température de l'eau, elles varient de 17,63 à $19,57{ }^{\circ} \mathrm{C}$. C'est une température favorable au développement des bactéries, des parasites, des larves de moustique et autres germes microbiens, arrivant à un maximum $19,57{ }^{\circ} \mathrm{C}$ au mois d'août. Elle présente une variation spatiale significative de l'amont vers l'aval (Figure 4), elle dépend des variations climatiques et non des stations étudiées (Aboulkacem et al., 2007). Les moyennes du pH varient entre 7,63 et 7,02 ne présentent pas de variation significative. Elles restent légèrement basiques à neutre et elles expriment l'alcalinité des différentes stations (amont-aval). La valeur la plus basse est enregistrée en station $\mathrm{S} 5$ où le $\mathrm{pH}=4,5$ est acide (Figure 4) car il y a rejet des margines pendant la période oléicole. Les valeurs minimales sont enregistrées au printemps où les conditions climatiques ne sont pas favorables pour la photosynthèse par contre les valeurs maximales sont enregistrées en été (Larif et al., 2012).

La dilution est favorisée plus entre les stations S3 et S4 par le mélange des eaux : courants, turbulence et action des marées. On estime que 90 à $99 \%$ des bactéries d'égout sont détruites après 48 heures de suspension dans l'eau et que leur nombre décroit avec la distance beaucoup plus rapidement que l'on pourrait s'y attendre du fait de la simple dilution (Maurin, 1974). D'autres auteurs ont confirmé le contraire, c'est-à-dire qu'il n'y a pas de corrélation entre les paramètres environnementaux et les bactéries (Kim et al., 2005).

On peut confirmer qu'il y a une évolution spatio-temporelle de la flore totale ainsi que des bactéries indicatrices de contamination fécale pour les sept stations en temps sec et de pluies (Figures 2 et 3 ). Cette évolution, en terme de concentrations, ne concorde pas avec celle obtenue dans la littérature vues les fortes précipitations au cours de l'année d'étude (2009-2010) et qui a provoqué le phénomène de dilution (Karrouch et al., 2009 ; Kacar, 2011).

Des résultats similaires en Algérie ont été enregistrés par d'autres études d'impact des eaux résiduaires superficielles par Hamaidi et al. (2009) ; et par l'étude de la contamination bactérienne dans le bassin de la seine à Paris (Servais et al., 2009).

La variation spatiale des nitrates de l'oued Boufekrane est due à la texture de la surface et à la nature lithologique des roches dont les résultats obtenus sont inférieurs à ceux de Bricha et al. (2007), est similaire à ceux de Belghiti et al. (2009). Les nitrates évoluent de façon inversement proportionnelle à la teneur en nitrites et au débit de l'oued Boufekrane d'après la Figure 2 (f). Le processus de dénitrification des nitrites en nitrates pourrait s'expliquer par la présence de bactéries autotrophes nitrosomonas et nitrobacters qui contribuent respectivement à l'oxydation de l'ammonium en nitrite et de l'oxydation des nitrites en nitrates. La consommation de l'oxygène dissous par les bactéries de l'amont vers l'aval est parmi l'un des processus (Pauwels, 1994; Issola et al., 2008).

Pour les germes pathogènes du genre Salmonelles, nous avons spécialement choisi au mois d'août les stations avals S3. S6 et S7 car ces dernières sont plus exposées à la pollution dans les eaux. On n'a pas pu les détecter malgré qu'il y ait contamination 
fécale. L'absence probable de ces germes à l'état viable et cultivable n'implique pas l'absence totale de ces dernières.

Il faut dire que la détection bactériologique et l'énumération quantitative de Salmonella dans les échantillons environnementaux est coûteuse, laborieuse, et fastidieuse, nécessitant jusqu'à 5 jours pour obtenir un résultat. D'une part leur présence en nombre relativement faible dans les eaux ainsi que leur difficulté d'y survivre ; d'autre part l'existence habituelle d'un nombre important de germes d'accompagnement, d'origine fécale (coliformes, streptocoques) ou non (Pseudomonas, Achromobacter, etc....) (Josefsen et al., 2007; Malorny et al., 2008). D'autres germes éventuellement pathogènes peuvent cohabiter avec les indicateurs de contamination fécale dénombrés, ce qui laisse à penser qu'il y a une corrélation entre les bactéries indicatrices de contamination fécale et les germes pathogènes genre Salmonelles (Lemarchand et Lebaron, 2003 ; Bonadonna et al., 2002; Horman et al., 2004), Ces constatations entraînent l'obligation d'utiliser des milieux d'enrichissements sélectifs, dans le but d'inhiber le développement des autres bactéries.

\section{Origine de la contamination}

Depuis plusieurs décennies, le rapport $\mathrm{R}=\mathrm{CF} / \mathrm{SF}$ (coliformes fécaux sur les streptocoques fécaux était utilisé comme un élément de premier ordre pour déterminer l'origine de la pollution fécale. Lorsque ce rapport $\mathrm{CF} / \mathrm{SF}$ est supérieur à 4 la pollution est essentiellement humaine (rejet des eaux usées) (Borrego et Romero, 1982). Lorsqu'il est inférieur à 0,7 , l'origine est animale. Dans ce cas, le bétail et en particulier les moutons, semble jouer un rôle prédominant dans la contamination de l'eau (Geldreich, 1976). Borrego et Romero (1982) ont envisagé différents cas pour expliquer l'origine de la contamination fécale en fonction du rapport de la valeur de R. Le Tableau 1 explique l'origine de la contamination de l'oued Boufekrane.
* En été pour la station (S1), (R étant compris $2>\mathrm{R}>1$ ) la contamination est incertaine. Pour les stations (S2, S3), R étant $<0,7$ la pollution est d'origine animale ce qui pourrait être expliqué par l'impact de rejet des abattoirs en aval de la commune de Boufekrane qui sont concentrées par le sang des animaux abattus et par les débris de panse (Belgghyti et al., 2009; Kacar, 2011). Pour les stations (S4, S5, S6, S7), (R étant compris $1>\mathrm{R}>0,7)$ la contamination est mixte à prédominance animale, on pourrait dire qu'en été les bergers font plus sortir leurs troupeaux qu'en été dans les pâturages pour brouter l'herbe dans les environs de l'oued Boufekrane.

* En automne pour la source S1 amont de la source, ( $\mathrm{R}$ étant compris $4>\mathrm{R}>2$ ) donc la contamination en septembre est exclusivement humaine dues aux rejets domestiques par la présence d'habitation dans les environs. Pour S5 $(\mathrm{R}<0,7)$ la pollution est d'origine animale et que pour les stations ( $\mathrm{S} 2, \mathrm{~S} 3, \mathrm{~S} 4, \mathrm{~S} 6, \mathrm{~S} 7)$ $2>\mathrm{R}>1$ est incertaine.

* En hiver pour la station S1, (4>R>2) se trouve une ferme à proximité caractérisé par une activité agricole et rejets domestiques, alors que pour les stations S2, S3, S4, S6 et S7, $(2>\mathrm{R}>1)$, la contamination est incertaine. Pour S5, $(\mathrm{R}<0,7)$ la contamination est d'origine animale, on pourrait l'expliquer par présence de bovins dans les pâturages à proximité de l'oued.

* Au printemps pour les stations S1, S3, $\mathrm{S} 4, \quad \mathrm{~S} 5$ et $\mathrm{S} 6,(1>\mathrm{R}>0,7)$; ceci peut être expliqué par la présence d'engrais phytosanitaires d'origine animal qu'utilise les agriculteurs sans compter le phénomène de lessivage des sols apporté par les fortes précipitations pendant le mois de mars. Pour S2, $(4>\mathrm{R}>2)$ on a l'urbanisation de la commune, ce qui fait qu'il y a des rejets domestiques. Pour S7, $(\mathrm{R}<0,7) \quad$ la contamination est principalement d'origine animale, à cause des rejets des abattoirs.

Selon Rodier (2009), les origines de la contamination des eaux ne proviennent pas d'une part exclusivement de l'origine humaine ou animale mais il y a aussi d'autre part les 
paramètres physico-chimiques qui contribuent à l'activité bactérienne comme la température et le pH (Chigbu et al., 2004).

\section{Analyse statistique}

L'Analyse en Composante Principale (ACP) a été également effectuée pour déceler la liaison entre les différentes variables, et variables-individus. Elle fait apparaître d'après le diagramme cartésien 88,27\% de l'information totale par les axes F1 et F2. L'axe F1 explique $71,41 \%$ de la variance qui est principalement dû à tous les paramètres bactériologiques mesurés, et que l'axe F2 représente $(16,86 \%)$ de la variance qui est localisé par la pollution en nitrates Figure 5.

En période de crue, l'analyse en composantes principales montre qu'il y a enrichissement de gauche vers la droite une contamination fécale et que les nitrates sont surtout présents dans les stations-saisons S1H1, S1A1, S2A2, S2H2, S3A3, S4H4, $\mathrm{S} 5 \mathrm{H} 5$ et $\mathrm{S} 5 \mathrm{~A} 5$, les concentrations en nitrates sont plus importantes et décroissent de l'amont vers l'aval (Larif et al., 2012). A l'observation, le diagramme cartésien confirme la corrélation entre la température, le $\mathrm{pH}$ et la population bactérienne des stations S1, S2, S3, S4, S6 et S7. Pendant la période oléicole, la station S5 est plus polluée en nitrate car il y a rejet des margines d'une huilerie et moins contaminée par les bactéries. Ces dernières ne résistent pas à la forte acidité des margines (Ranalli et al., 1991 ; Larif et al., 2012). Les stations S5 sont situées à gauche du diagramme cartésien, ce qui confirme l'absence de paramètres bactériologiques. En aval la pollution bactérienne des stations (S6A6, S7H7) est véhiculée par les fortes pentes des reliefs au niveau de l'oued Boufekrane, elle provient du lessivage des sols (Korfalis et Davies, 2003).

En période d'étiage, les nitrates sont moins présents dans les stations-saisons S3E3, S5E5, S4E4, S6P6 et S7E7 mais sont plus contaminées par la présence de bactéries hétérotrophes. Par conséquent, la recherche et le dénombrement des coliformes fécaux est un examen proposé en raison d'une concordance statistique entre leurs présences et l'existence d'une contamination fécale quasi certaine (Rodier et al., 1996). Alors que la Flore Mésophile Aérobie Totale est seulement un indicateur microbiologique qui nous permet d'évaluer la charge bactérienne globale présente sur la surface de l'oued Boufekrane. L'interaction entre les variables bactériologiques considérées dans cette étude montre que le suivi de l'une d'entre elle, suffirait pour la surveillance de la qualité bactériologique (Rodier, 2009).

\section{Conclusion}

Les résultats d'analyses bactériologiques obtenus ont montré que la pollution présente une différence significative entre les différentes stations de prélèvement qu'en milieu très pollués et entre les périodes de crues et les périodes d'étiages et la durée d'ensoleillement.

L'origine de la contamination transmise par les cours d'eaux, confirme l'impact de l'intensification de l'agriculture ainsi que par les rejets domestiques et industriels.

L'analyse en composante principale a confirmé qu'en amont les eaux de l'oued Boufekrane sont caractérisées par une forte pollution en nitrate pendant la période de crue et moins en contamination fécale, alors qu'en aval les eaux de l'oued sont caractérisées plus par une contamination fécale et faible en pollution nitrate. Bien que les eaux usées transportent de nombreux micro-organismes, parmi lesquels certains sont peu ou pas pathogènes, très peu de stations d'épuration sont cependant aujourd'hui équipées de traitements spécifiquement conçus pour éliminer les microorganismes (traitement de désinfection). Il faudrait aussi prendre des mesures restrictives sur les déversements industriels en leur imposant la loi pollueur/payeur.

\section{REFERENCES}

Aboulkacem A, Chahlaoui A, Soulaymani A, Rhazifilali F, Benali D. 2007. Etude comparative de la qualité bactério logique des eaux des oueds Boufekrane et Ouislane à la 
traversée de la ville de Meknès (Maroc). Rev. Microbiol. Ind. San. Environ., 1: 1022.

Achak M, Ouazzani N, Yaacoubi A, Mandi L. 2008. Traitement des margines par coagulation-floculation. Revue des Sciences de l'Eau, 21(1): 53-67.

Belghyti D, El guamri Y, Ztit G, Uahidi ML, Joti MB, Harchrass A, Amghar H, Bouchouate O, EL Kharrim K, Bounouira H. 2009. Caractérisation physicochimique des eaux usées d'abattoirs en vue de la mise en œuvre d'un traitement adéquat: cas de Kénitra au Maroc. Afrique Sciences, 05(2): 199-216

Blanchoud H, Farrugia F, Mouchel JM. 2004. Pesticide uses and transfers in urbanised catchments. Chemosphere, 55: 905-913.

Blanchoud H, Moreau-Guigon E, Farrugia F, Chevreuil M, Mouchel JM. 2007. Contribution by urban and agricultural pesticide uses to water contamination at the scale of the Marne watershed. Sci. Total Environ., 375: 168-179.

Bonadonna L, Briancesco R, Ottaviani M, Veschetti E. 2002. Occurrence of Cryptosporidium oocysts in sewage effluents and correlation with microbial, chemical, and physical water variables. Environ. Monit. Assess., 75: 241-252.

Borrego AF, Romero P. 1982. Study of the microbiological pollution of a Malaga littoral area II. Relationschip between fecal coliforms and fecal streptococci. VIè Journée Étude Pollutions, Cannes, France, 561-569.

Bousaab H, Nassif N, EL Samrani AG, Daoud R, Medawar S, Ouaini N. 2007. Suivi de la qualité bactériologique des eaux de surface (rivière Nahr Ibrahim, Liban). Revue des Sciences de l'Eau / Journal of Water Science, 20(4): 341-352.

Botta F, Fauchon N, Blanchoud H, Chevreuil M, Guery B. 2012. Phyt'Eaux Cités: Application and validation of a programme to reduce surface water contamination with urban pesticides. Chemosphere, 86: 166-176.

Chigbu P, Gordon S, Strange T. 2004. Influence of inter-annual variations in climatic factors on fecal coliform levels in Mississippi sound. Water Res., 38: 4341-4352.

Denis M, Tanguy M, Chidaine B, Laisney MJ, Megraud F, Fravalo P. 2011. Description and sources of contamination by Campylobacter spp. of river water destined for human consumption in Brittany, France. Pathologie Biologie, 59: 256-263.

Fagrouch A, Berrahou, EL Halouani H. 2011. Impact d'un effluent urbain de la ville de Taourirt sur la structure des communautés de macroinvertébrés de l'oued Za (Maroc oriental). Revue des Sciences de l'Eau / Journal of Water Science, 24(2): 87-101.

Figarella J, Leyral G, Terret M. 2001. Microbiologie Générale et Appliquée. Edit. Jacques Lanore, 285.

Genin B, Chauvin C, Menard F. 2003. Cours d'Eau et Indices Biologiques, Pollutions Méthodes IGBN (2nd edn). Educagri.

George I, Servais P. 2002. Sources et Dynamique des Coliformes dans le Bassin de la Seine. Centre National de la Recherche Scientifique: Paris, France; 46.

Hadou-Sanoun G, Arab A, Lek-Ang S, Sovan Lek S. 2012. Impact de Ligula intestinalis (L.1758) (Cestode) sur la croissance de Barbus setivimensis (Cyprinidae) dans un système lacustre Algérien. $C$. $R$. Biologies, 335: 300-309.

Hamaidi F, Hamaidi MS. 2009. Recherche des indicateurs bactériens de contaminations fécale dans les eaux de barrage Lakhal (Bouira Algérie). Rev. Microbiol. Ind. San. Environn, 3(1): 76-95.

Hartemann P. 2004. Contamination des eaux en milieu professionnel Water contamination in working place. EMCToxicologie Pathologie, 1: 63-78. 
Hebert S, Legare S. 2000. Suivi de la qualité des rivières et petits cours d'eau. Direction du suivi de l'état de l'environnement, Ministère de l'Environnement, Québec, envirodoq, $\mathrm{N}^{\mathrm{O}}$ ENV-2001-0141, rapport $\mathrm{N}^{\circ}$ QE-123, 24 p. et 3 annexes.

Horman A, Rimhanen-Finne R, Maunula L, Von Bonsdorff C-H, Torvela N, Heikinheimo A, Hanninen M-L. 2004. Campylobacter spp., Giardia spp., Cryptosporidium spp., Noroviruses, and indicator organisms in surface water in southwestern Finland, 2000-2001. Appl. Environ. Microbiol., 70 (1):87-95.

Issola Y, Kouassi AM, Dongui BK, Jean B. 2008. Caractéristiques physico-chimiques d'une lagune côtière tropicale : lagune de Fresco (Côte d'Ivoire). Afrique Science, 04: 368-393.

Josefsen MH, Krause M, Hansen F, Hoorfar J. 2007. Optimization of a 12-Hour Taq Man PCR based method for detection of Salmonella bacteria in meat. Appl. Environ Microbiol., 73: 3040-3048.

Kacar A. 2011. Analysis of spatial and temporal variation in the levels of microbial fecal indicators in the major rivers flowing into the Aegean Sea, Turkey. Ecological Indicators, 11: 13601365.

Karrouch L, Chahlaoui A. 2009. Bioévaluation de la qualité des eaux de l'oued Boufekrane (Meknès, Maroc). Biomatec Echo, 3(6): 6-17.

Kim G, Choib E, Lee D. 2005. Diffuse and point pollution impacts on the pathogen indicator organism level in the Geum River, Korea. Sci. Total Environ., 350: 94-105.

Korfali S, Davies B. 2003. A comparison of metals in sediment and water in the river Nahr-Ibrahim, Lebanon: 1996 and 1999. Environ. Geochem. Health, 25: 41-50.

Larif M, Soulaymani A, Hnach M, EL Midaoui A. 2012a. Olive Wastewaters' Impact on Oued Boufekrane in Meknes-
Tafilalet. American Journal of Environmental Sciences, 8(3): 236-240.

Larif M, Adad A, Hmammouchi R, Idrissi Taghki A, Soulaymani A, Elmidaoui A, Bouachrine M, Lakhlifi T. 2013. Biological activities of triazine derivatives. Combining DFT and QSAR Results. Arabian Journal of Chemistry, http://dx.doi.org/10.1016/j.arabjc.2012.12 .033 .

Lemarchand K, Mason L, Brousseau R. 2004. Molecular biology and DNA microarray technology for microbial quality monitoring of water. Crit. Rev. Microbiol., 30: 145-172.

Lipp EK, Rodriguez-Palacios C, Rose JB. 2001. Occurrence and distribution of the human pathogen Vibrio vulnificus in a subtropical Gulf of Mexico estuary. Hydrobiologia, 460: 165-17.

Malorny B, Löfström C, Wagner M, Krämer N, Hoorfar J. 2008. Enumeration of Salmonella bacteria in food and feed samples by real-time PCR for quantitative microbial risk assessment. Appl. Environ. Microbiol., 74:1299-304.

Maurin C. 1974. La conchyliculture française: le milieu naturel et ses variations (première partie). Institut Scientifique et Technique des Pêches Maritimes, Nantes. P112 -117.

Oulkheir S, Ounine K, EL Haloui N, Ikko L, Bricha S, Sabhi Y, Attrassi B. 2007. Etude de la qualité physico-chimique et bactériologique de la nappe phréatique M'nasra (Maroc). Afrique Science, 03(3): 391- 404.

Pauwels H. 1994. Natural denitrification in groundwater in the presence of pyrite; preliminary results obtained at Naising (Brittany, France). Mineralogical Magazine, 58(A): 696-697.

PNUE / OMS. 1977. Recommandation pour la surveillance sanitaire des zones côtières à usage récréatif et des zones conchylicoles. Bureau régional de l'OMS pour l'Europe, Copenhague, 168p. 
Ranalli A. 1991. L'effluent des huileries d'olive: proposition en vue de son épuration. Références aux normes italiennes en la matière, première partie, Olivia, 37: 30-39.

Rodier J, Legube B, Merlet N. 2009. L'Analyse de l'Eau, Eaux Naturelles, Eaux Résiduaires, Eaux de Mer (9th edn). Dunod : Paris.

Rodier J, Bazin C, Broutin JP, Chambon P, Champsaur H, Rodier L. 1996. L'Analyse de l'Eau, Eaux Naturelles, Eaux Résiduaires, Eaux de Mer (8th edn). Dunod : Paris ; 1384.

Servais P. 2009. La contamination microbienne de la seine. Janvier: 978-2918251-08-8.

XU S, Jinhua L, Lingxin C. 2011. Molecularly imprinted polymers by reversible addition-fragmentation chain transfer precipitation polymerization for preconcentration of atrazine in food matrices. Talanta, 85: 282-289.

Toilabiya L, Soulaymani A, Benali D, Hami H, Ouammi L, Mokhtari A, Rhalem N, SoulaymanI R. 2012. Profil épidémiologique des intoxications dans la région de Tanger-Tétouan au Maroc. Antropo., 26: 49-57.

Wittmer IK, Bader HP, Scheidegger R, Singer H, Lück A, Hanke I, Carlsson C, Stamm, C. 2010. Significance of urban and agricultural land use for biocide and pesticide dynamics in surface waters. Water Res., 44(9): 2850-2862. 